\title{
Multiresolution Application of Artificial Intelligence in Digital Pathology for Prediction of Positive Lymph Nodes From Primary Tumors in Bladder Cancer
}

Stephanie A. Harmon, PhD ${ }^{1,2}$; Thomas H. Sanford, MD ${ }^{2,3}$; G. Thomas Brown, MD, PhD ${ }^{2,4}$; Chris Yang ${ }^{1}$; Sherif Mehralivand, MD ${ }^{1}$; Joseph M. Jacob, MD³; Vladimir A. Valera, MD, PhD ${ }^{5}$; Joanna H. Shih, PhD ${ }^{6}$; Piyush K. Agarwal, MD ${ }^{5}$; Peter L. Choyke, MD ${ }^{1}$; and Baris Turkbey, MD ${ }^{1}$

PURPOSE To develop an artificial intelligence (AI)-based model for identifying patients with lymph node (LN) metastasis based on digital evaluation of primary tumors and train the model using cystectomy specimens available from The Cancer Genome Atlas (TCGA) Project; patients from our institution were included for validation of the leave-out test cohort.

METHODS In all, 307 patients were identified for inclusion in the study (TCGA, $n=294$; in-house, $n=13$ ). Deep learning models were trained from image patches at 2.5x, 5x, 10x, and 20x magnifications, and spatially resolved prediction maps were combined with microenvironment (lymphocyte infiltration) features to derive a final patient-level Al score (probability of LN metastasis). Training and validation included 219 patients (training, $n=146$; validation, $n=73$ ); 89 patients (TCGA, $n=75$; in-house, $n=13$ ) were reserved as an independent testing set. Multivariable logistic regression models for predicting LN status based on clinicopathologic features alone and a combined model with Al score were fit to training and validation sets.

RESULTS Several patients were determined to have positive LN metastasis in TCGA ( $n=105 ; 35.7 \%$ ) and inhouse $(n=3 ; 23.1 \%)$ cohorts. A clinicopathologic model that considered using factors such as age, T stage, and lymphovascular invasion demonstrated an area under the curve (AUC) of $0.755(95 \% \mathrm{Cl}, 0.680$ to 0.831$)$ in the training and validation cohorts compared with the cross validation of the Al score (likelihood of positive LNs), which achieved an AUC of $0.866(95 \% \mathrm{Cl}, 0.812$ to $0.920 ; P=.021)$. Performance in the test cohort was similar, with a clinicopathologic model AUC of $0.678(95 \% \mathrm{Cl}, 0.554$ to 0.802$)$ and an $\mathrm{Al}$ score of $0.784(95 \% \mathrm{Cl}, 0.702$ to $0.896 ; P=.21$ ). In addition, the Al score remained significant after adjusting for clinicopathologic variables $\left(P=1.08 \times 10^{-9}\right)$, and the combined model significantly outperformed clinicopathologic features alone in the test cohort with an AUC of $0.807(95 \% \mathrm{Cl}, 0.702$ to $0.912 ; P=.047)$.

CONCLUSION Patients who are at higher risk of having positive LNs during cystectomy can be identified on primary tumor samples using novel Al-based methodologies applied to digital hematoxylin and eosin-stained slides.

JCO Clin Cancer Inform 4:367-382. @ 2020 by American Society of Clinical Oncology

ASSOCIATED CONTENT

Appendix

Author affiliations

and support

information (if

applicable) appear at

the end of this

article.

Accepted on February

26, 2020 and

published at

ascopubs.org/journal/

cci on April 24, 2020:

DOI https://doi.org/10.

1200/CCI.19.00155

\section{INTRODUCTION}

Risk stratification is essential in determining the optimal treatment strategy for patients with bladder cancer. Bladder cancer is broadly divided into two risk groups based on invasion of the muscular wall (muscularis propria) of the bladder: nonmuscle invasive bladder cancer (NMIBC) and muscle invasive bladder cancer (MIBC). In general, NMIBC is associated with a relatively favorable long-term prognosis compared with MIBC, which carries a worse prognosis with up to $50 \%$ of patients developing disease recurrence. ${ }^{1,2}$ For patients diagnosed with localized $\mathrm{MIBC}$, the mainstay of therapy is radical cystectomy
(RC) with pelvic lymph node (LN) dissection. ${ }^{3}$ Although RC achieves negative margins in up to $96 \%$ of patients, ${ }^{4}$ recurrence rates of up to $50 \%$ during followup suggest that there are likely microscopic extravesical tumor deposits present at the time of surgical resection in a substantial portion of patients. ${ }^{5}$ To reduce recurrences after surgery, neoadjuvant chemotherapy (NAC) has been administered using a fourdrug regimen containing methotrexate, vincristine, doxorubicin, and cisplatin. ${ }^{6}$ Although there have been attempts to further risk stratify MIBC on the basis of adverse or variant histology and clinical staging, ${ }^{7}$ there has not yet been widespread adoption of further risk 


\section{CONTEXT}

\section{Key Objective}

To evaluate whether the histologic appearance and features of primary tumors can be used to predict the metastatic potential of disease at the time of surgical intervention using advanced artificial intelligence techniques. Here we investigate whether digital pathology specimens from primary tumors from RC can be used to predict whether the patient will have positive LN findings.

\section{Knowledge Generated}

Convolutional neural networks are able to learn distinct features within tumor specimens which, combined with microenvironmental features, are associated with increased likelihood of positive LNs, and these features are independent of known clinicopathologic prognostic features.

\section{Relevance}

MIBC suffers from a lack of robust clinical and pathologic variables for stratification of patients with a high risk of recurrence after surgery; the algorithm presented here could serve as an additional prognostic biomarker in this setting.

stratification approaches to cluster patients with MIBC into higher and lower risk groups for selection of NAC before surgical intervention. These pathologic features are typically identified on tissue acquired from transurethral resection of bladder tumor (TURBT) procedures and have highly variable concordance with final RC findings in the literature. $^{8}$ Therefore, it would be ideal if prognostic markers were developed that do not suffer from interobserver variability and reflect the nature of final pathology features at RC.

Traditionally, pathology slides undergo qualitative interpretation and assessment by the pathologist to generate a clinical report from which a small fraction of the information is used for clinical risk evaluation. Advances in artificial intelligence (AI) techniques in computer vision using deep convolutional neural networks (CNNs) have allowed for the development of prognostic models that use digital images of pathology slides that can evaluate the entirety of the available image. ${ }^{9}$ The theoretical hypothesis of this work is that the histologic appearance of the primary tumor contains information related to the metastatic potential of that tumor. In this study, we tested this hypothesis by developing an Al-based model for identifying patients with LN metastasis based solely on evaluation of primary tumors, and the model was trained by using digitized cystectomy specimens available from The Cancer Genome Atlas (TCGA) Project and validated in a separate test cohort that included patients from our institution.

\section{METHODS}

\section{Patient Population and Slide Review}

All available digital pathology slides (457 slides from 386 patients) derived from cystectomy specimens that were stained with hematoxylin and eosin (H\&E) were downloaded from the National Cancer Institute Genomic Data Commons Data Portal (https://portal.gdc.cancer.gov/; accession date: November 5, 2018). The presence of LN metastasis (positive or negative), number of excised LNs, and presence of variant histology on routine H\&E examination were collected from surgical cystectomy pathology reports available at the cBioPortal (http://www.cbioportal. org). Clinical, demographic, and patient outcome data were accessioned from publicly available TCGA outcomes resources reported in Liu et al. ${ }^{10}$ Download instructions for reproducibility are provided in the Appendix. T stages reported in this study refer to pathologic $T$ stage from cystectomy. Of the variant histologies represented in the TCGA cohort (Table 1), the following were classified as high risk: neuroendocrine, micropapillary, and plasmacytoid. A board-certified pathologist (G.T.B.) reviewed all diagnostic slides for the presence and extent of tumor and manually annotated tumor regions within the slide. Ninety-two patients were excluded from this study because of missing or low-quality data (Appendix Fig A1). The number of slides that were publicly available from the TCGA cohort varied; a majority of patients had only one representative slide of the tumor specimen (median, one slide per patient; range, 1-8 slides per patient). At model inference and evaluation, only the first diagnostic slide for each patient (denoted DX1 in the TCGA cohort) was used in analysis, consistent with previous studies using this data set. ${ }^{11}$

For testing on an external cohort, 43 patients were identified from our local institution who underwent radical cystectomy between 2013 and 2019. Of these patients, 30 were excluded on the basis of the aforementioned exclusion criteria, availability of diagnostic slides for review and digitization, or those undergoing more than two types of neoadjuvant therapy. This study is compliant with local institutional review board and Health Insurance Portability and Accountability Act (HIPAA) guidelines. Although the trained algorithm was applied to all slides from an individual patient, only a single slide with the largest tumor burden was used for statistical analysis to remain consistent with the TCGA data set. 
TABLE 1. Clinical and Pathologic Characteristics of TCGA and In-House Patients

TCGA

\section{Training and Validation}

Cohorts

Test Cohort

In-House Cohort

\begin{tabular}{|c|c|c|c|c|c|c|}
\hline Characteristic & No. & $\%$ & No. & $\%$ & No. & $\%$ \\
\hline \multicolumn{7}{|l|}{ Sex } \\
\hline Male & 161 & 74 & 54 & 72 & 12 & 92 \\
\hline Female & 58 & 26 & 21 & 28 & 1 & 8 \\
\hline \multicolumn{7}{|l|}{ Race } \\
\hline White & 180 & 82 & 63 & 84 & 10 & 77 \\
\hline African American & 11 & 5 & 6 & 8 & 1 & 8 \\
\hline Asian & 23 & 11 & 6 & 8 & 1 & 8 \\
\hline NA & 5 & 2 & - & & 1 & 8 \\
\hline \multicolumn{7}{|l|}{ Histologic grade } \\
\hline Low & 12 & 5 & 2 & 3 & - & \\
\hline High & 206 & 94 & 73 & 97 & 13 & 100 \\
\hline Unknown & 1 & $<1$ & - & & - & \\
\hline \multicolumn{7}{|l|}{ Smoking history } \\
\hline Lifelong nonsmoker & 61 & 28 & 23 & 30 & 4 & 31 \\
\hline Current smoker & 45 & 21 & 12 & 16 & 3 & 23 \\
\hline Reformed smoker & 105 & 48 & 37 & 49 & 4 & 31 \\
\hline NA & 8 & 4 & 3 & 4 & 2 & 15 \\
\hline \multicolumn{7}{|l|}{ Pathologic T stage } \\
\hline $\mathrm{T} 2$ & 66 & 30 & 22 & 29 & 2 & 15 \\
\hline T3 & 119 & 54 & 41 & 55 & 3 & 23 \\
\hline $\mathrm{T} 4$ & 34 & 16 & 12 & 16 & 8 & 62 \\
\hline \multicolumn{7}{|l|}{ Variant histology } \\
\hline Micropapillary & 7 & 3 & 2 & 3 & 1 & 8 \\
\hline Neuroendocrine & 3 & 1 & - & & - & \\
\hline Plasmacytoid & 1 & $<1$ & - & & - & \\
\hline Sarcomatoid & 6 & 3 & 1 & 1 & - & \\
\hline Squamous & 46 & 21 & 10 & 13 & 5 & 38 \\
\hline Other & 7 & 3 & 1 & 1 & 1 & 8 \\
\hline None specified & 135 & 62 & 56 & 75 & 5 & 38 \\
\hline \multicolumn{7}{|l|}{ LVI } \\
\hline Not present & 74 & 34 & 33 & 44 & 5 & 38 \\
\hline Present & 106 & 48 & 33 & 44 & 8 & 62 \\
\hline NA & 39 & 18 & 9 & 12 & - & \\
\hline \multicolumn{7}{|l|}{ LN status } \\
\hline Negative (NO) & 141 & 64 & 48 & 64 & 10 & 77 \\
\hline Positive (N1/N2) & 78 & 36 & 27 & 36 & 3 & 23 \\
\hline Median No. of LNs examined (range) & $21(1-170)$ & & $17(2-83)$ & & $27(7-38)$ & \\
\hline Median No. of positive LNs (range) & $3(1-97)$ & & $2(1-16)$ & & $2(1-7)$ & \\
\hline \multicolumn{7}{|l|}{ Overall survival } \\
\hline Alive & 122 & 56 & 35 & 47 & - & \\
\hline Dead & 97 & 44 & 40 & 53 & - & \\
\hline
\end{tabular}

(Continued on following page) 
TABLE 1. Clinical and Pathologic Characteristics of TCGA and In-House Patients (Continued)

TCGA

\begin{tabular}{|c|c|c|c|c|c|c|}
\hline \multirow[b]{2}{*}{ Characteristic } & \multicolumn{2}{|c|}{$\begin{array}{c}\text { Training and Validation } \\
\text { Cohorts }\end{array}$} & \multicolumn{2}{|c|}{ Test Cohort } & \multicolumn{2}{|c|}{ In-House Cohort } \\
\hline & No. & $\%$ & No. & $\%$ & No. & $\%$ \\
\hline \multicolumn{7}{|c|}{ Disease-specific survival } \\
\hline Alive & 145 & 66 & 47 & 63 & - & \\
\hline Dead & 67 & 31 & 24 & 32 & - & \\
\hline NA & 7 & 3 & 4 & 5 & - & \\
\hline
\end{tabular}

NOTE. All pathologic variables (grade, T stage, variant histology, lymphovascular invasion) are reported from the cystectomy review. Abbreviations: LN, lymph node; LVI, lymphovascular invasion; NA, not applicable; TCGA, The Cancer Genome Atlas.

\section{Image-Based Classification Pipeline}

The entire image processing and classification pipeline is shown in Figure 1. TCGA patients were randomly assigned to the training cohort ( $50 \%[n=146])$, the validation cohort (25\% [ $n=73])$, or the testing cohort ( $25 \%[n=75])$, and an in-house cohort was added to the testing set (Fig 1B). Given the large input size of digital pathology images, only regions containing tumor from the bladder cystectomy specimens were used. These regions were evenly divided into smaller image patches for deep learning-based classification. Multiresolution probability maps were constructed to allow for spatially resolved predictions from all deep learning models and were combined with microenvironmental features (burden and spatial organization of lymphocyte infiltration) to derive a final patient-level Al score, which represents the likelihood of a patient having positive LN status at the time of surgery. These procedures were then applied to the patients in the test cohort. Full details are provided in the Appendix.

Stain normalization and patch extraction. For each digital slide, images were read in at a 4:1 ratio from apparent 40x magnification digital images to allow for whole-slide image processing. ${ }^{12,13}$ Image preprocessing included stain normalization, background artifact removal, and stain deconvolution using previously published methods. ${ }^{14,15}$ Region selection was determined by fitting a minimum number of non-overlapping regions that fully contained pathologist annotations. Within each region, 1, 4, 16, and 64 patches were mapped corresponding to $300 \times 300$ pixel images at magnifications of $2.5 \times, 5 \times, 10 \times$, and $20 \times$, respectively. The same coordinates were used to guide extraction of 576 images of $100 \times 100$ pixels at an effective $20 \times$ resolution from the original $\mathrm{H \& E}$ images for estimating the presence of lymphocyte infiltration using the algorithm of Saltz et al. ${ }^{11,16}$

Patch-based classification learners. Four different models using ResNet-101 architecture were trained from patches at $2.5 x, 5 x, 10 x$, and $20 x$ magnifications, respectively, using fast.ai library (https://github.com/fastai/fastai) with weighted cross-entropy loss. Augmentation techniques that included orientation and intensity were applied randomly during training to reduce overfitting patient-specific and slide-specific characteristics. Final training conditions are included in the Appendix and Appendix Table A1. At deployment, the corresponding output (probability of being LN positive) was saved for each patch image at every resolution. For lymphocyte classification, publicly available code and model weights were downloaded from Saltz et $a{ }^{11}{ }^{11}$ and consistency in results from our preprocessing methods compared with those in the original study were confirmed (Appendix Fig A2). This model was applied to the slides for all patients, and the probability of containing a lymphocyte was saved for each patch.

Post-processing and patient-based classification learner. Given that all patches were extracted within known coordinates, the final result is a multiresolution prediction map in which every pixel represents the average probability of LN status across deep learning models. A simplified majority vote score was calculated as the burden of tumor patches with probability greater than $50 \%$ for $L N$ positivity across individual models. The final score was derived from high-risk clusters (ie, high probability) that were identified with density-based spatial clustering of applications with noise (DBSCAN) using starting conditions derived from varying probability thresholds and extraction of metrics related to size, number, density, and distance between clusters and lymphocytes (Fig 1A). A full description is presented in the Appendix and Appendix Table A2. An adaptive boosting classification ensemble of decision trees (AdaBoost ${ }^{17}$ ) was fit using 10-fold cross validation within combined training and validation cohorts (Fig 1). Variable selection $^{18}$ and training conditions are detailed in the Appendix. Classification performance was determined by cross-validation out-of-bag estimates for the training and validation cohorts and was then applied to testing cohorts. Classification outputs were saved as binary (yes/no LN positive) and continuous (range, $0-1$ ), which is referred to as the Al score hereafter. All post-processing and AdaBoost classifications were developed in MATLAB (R2018b; www. mathworks.com). ${ }^{18}$

\section{Statistical Analysis}

The accuracy of Al-based models was reported for patchlevel and patient-level results in each cohort. The final Al 


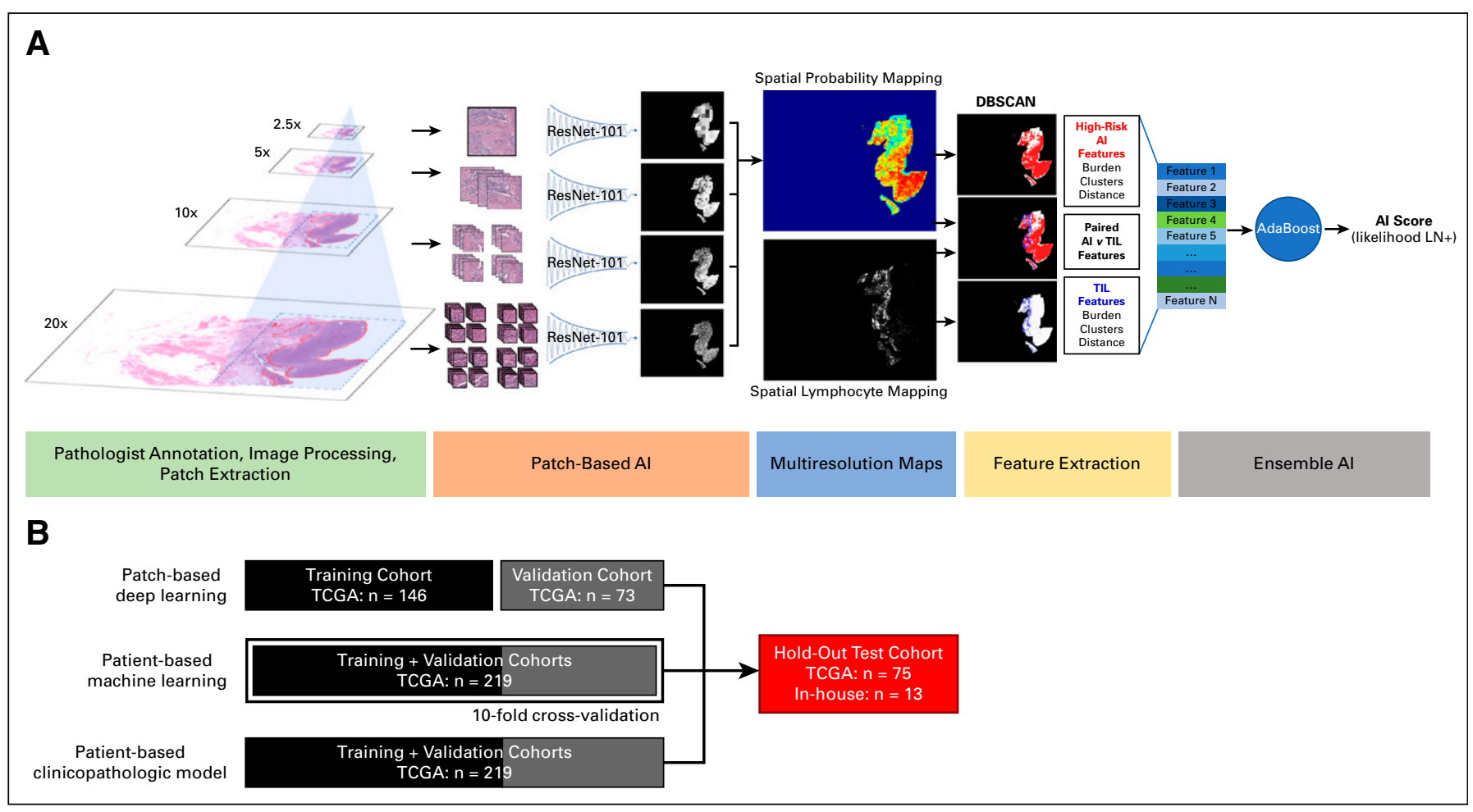

FIG 1. (A) Deep learning workflow. After tumor regions were annotated by a pathologist, patches of equal size were extracted across four resolutions for training the patch-based convolutional neural network application (ResNet-101). Spatial referencing of patch location and relative size (compared with highest resolution) allowed for construction of multiresolution probability maps from convolutional neural network outputs. These probability maps were used to define high-risk regions based on various probability thresholds as input to density-based spatial clustering of applications with noise (DBSCAN) and combined with lymphocyte infiltration maps for input to a machine learning-based classifier. (B) Training, validation, and testing schema. For patch-based deep learning, training and validation cohorts were kept separate. For patient-based machine learning, training and validation sets were combined, and 10fold cross validation was used in model development. For clinicopathologic-based logistic regression analysis, training and validation cohorts were combined. Al, artificial intelligence; LN, lymph node; TCGA, The Cancer Genome Atlas; TIL, tumor-infiltrating lymphocytes.

score, reflecting the probability of $L N$ positivity (range, $0-1$ ), was used to derive the area under the curve (AUC). The accuracy, specificity, sensitivity, and positive predictive value reported in the combined training and validation sets are the binary out-of-bag predictions in 10-fold cross validation.

A multivariable logistic regression model for prediction of LN status based on clinicopathologic features was developed after univariable assessment. Variables with known clinical significance and those with significant association with LN status were fit using backward Akaike information criterion. A combined model that included the Al score was added to the multivariable model and fit to the training and validation sets. Both multivariable models were applied to test sets. The classification performance of the logistic regression model was reported by the AUC and evaluated using the pROC package in $\mathrm{R}$.

All reported $P$ values are two-sided, and $P<.05$ was used to determine statistical significance. Statistical analyses were conducted using R software version 3.4.1 (http:// www.r-project.org/). All code used for model development and statistical analysis is publicly available at https:// github.com/NIH-MIP/BLCA_LNprediction.git.

\section{RESULTS}

The final cohort consisted of 294 patients from the TCGA cohort and 13 patients from the in-house cohort; 105 patients from the TCGA cohort and 3 patients from the in-house cohort had positive LN metastasis at the time of surgery. Patient demographics are listed in Table 1. There were no statistically significant differences between TCGA patient samples used for the training and validation cohorts compared with those left out in the test cohort (Appendix Table A3). For multivariable analysis, a final model was constructed by using the following input variables: age, disease stage, presence of high-risk histology, lymphovascular invasion (LVI), and number of LNs excised (Table 2). After backward Akaike information criterion selection, the final multivariable model included age, T stage, and the presence of LVI. Using this model for predicting LN status resulted in an AUC of $0.755(95 \% \mathrm{Cl}, 0.680$ to 0.831$)$ in the combined training and validation cohorts (Table 3 ). When applied to the testing cohort, the performance of the multivariable model decreased to an AUC of $0.678(95 \% \mathrm{Cl}, 0.554$ to 0.802).

By combining features extracted from both multiresolution prediction maps and lymphocyte infiltration maps (Fig 1A), 
TABLE 2. Logistic Regression Model for Association of Clinical and Pathologic Variables With LN Status in the Training and Validation Cohorts

Univariable

Multivariable

\begin{tabular}{|c|c|c|c|c|c|c|}
\hline \multirow[b]{2}{*}{ Characteristic } & & \\
\hline & OR & $95 \% \mathrm{Cl}$ & $P$ & OR & $95 \% \mathrm{Cl}$ & $P$ \\
\hline Age, years & 1.03 & 1.01 to 1.06 & .012 & 1.03 & 1.00 to 1.06 & .106 \\
\hline \multicolumn{7}{|l|}{ Sex } \\
\hline Female & Reference & & & & & \\
\hline Male & 1.07 & 0.57 to 2.03 & .834 & & & \\
\hline \multicolumn{7}{|l|}{ Race } \\
\hline White & Reference & & & & & \\
\hline Asian & 0.22 & 0.05 to 0.67 & .018 & & & \\
\hline African American & 0.33 & 0.05 to 1.31 & .159 & & & \\
\hline \multicolumn{7}{|l|}{ Smoking status } \\
\hline Current & Reference & & & & & \\
\hline Reformed & 2.06 & 0.96 to 4.67 & .071 & & & \\
\hline Nonsmoker & 1.51 & 0.64 to 3.67 & .355 & & & \\
\hline \multicolumn{7}{|l|}{ Stage } \\
\hline T2 & Reference & & & Reference & & \\
\hline T3/T4 & 3.01 & 1.55 to 6.18 & .002 & 2.53 & 1.14 to 5.97 & .027 \\
\hline \multicolumn{7}{|l|}{ Grade } \\
\hline High & Reference & & & & & \\
\hline Low & $1.07 \times 10^{-7}$ & 0 to $3.1 \times 10^{14}$ & .982 & & & \\
\hline \multicolumn{7}{|l|}{ High-risk variant histology } \\
\hline Not present & Reference & & & & & \\
\hline Present & 5.26 & 1.47 to 24.6 & .017 & & & \\
\hline \multicolumn{7}{|l|}{ LVI } \\
\hline Not present & Reference & & & Reference & & \\
\hline Present & 6.16 & 2.96 to 13.9 & $3.42 \times 10^{-6}$ & 5.41 & 2.55 to 12.4 & $2.50 \times 10^{-5}$ \\
\hline No. of excised LNs examined & 1.01 & 1.00 to 1.02 & .113 & & & \\
\hline
\end{tabular}

NOTE. Each variable is reported in the univariable analysis. The final multivariable model included age, T stage, and lymphovascular invasion $(\mathrm{LVI})$.

Abbreviations: LN, lymph node; OR, odds ratio.

the Al score (likelihood of LN positivity) achieved an AUC of $0.866(95 \% \mathrm{Cl}, 0.812$ to 0.920$)$ in the training and validation cohorts and $0.784(95 \% \mathrm{Cl}, 0.702$ to 0.896$)$ in the test cohort (Fig 2; Table 3). The Al score remained significant when it was included in the multivariable logistic regression model with clinicopathologic variables (odds ratio, $6.36 ; 95 \% \mathrm{Cl}$, 3.66 to $12.1 ; P=1.08 \times 10^{-9}$ ), which significantly outperformed clinicopathologic features alone in the hold-out test cohort with an AUC of 0.807 versus $0.678(P=.047$; Table 3). On its own, the Al model achieved $82.6 \%$ specificity with $71.4 \%$ positive predictive value for the combined training and validation cohorts (cross-validated out-of-bag predictions) and $84.5 \%$ specificity with $66.7 \%$ positive predictive value in the test cohort (Appendix Table A4). Specifically, accuracy was $84.6 \%$ on the patient level and accuracy was $71.1 \%$ on the slide level within the in-house test cohort.

Slide images from a representative true-positive patient from the test cohort are shown in Figure 3. In assessing the relationship of the Al score to known adverse pathologic features, binary classification by Al score was significantly associated with the presence of high-risk variant histology in both training and testing cohorts $\left(\chi^{2}\right.$ test $P=.019$ and $P=$ .044 , respectively). In the training set, the Al score binary classification was significantly associated with LVI $\left(\chi^{2}\right.$ test $P=.0005)$, likely reflecting the correlation of each to $L N$ status. In the test set, no association between Al score and LVI was observed ( $\chi^{2}$ test $P=1$ ).

Results pertaining to patch-based training and performance at the individual magnification level and at combined magnification levels are presented in the Appendix. The overall Al score generalized better in the test cohort compared with the simplified majority vote score in which AUC was $0.762(95 \% \mathrm{Cl}, 0.662$ to $0.862 ; P=.24)$ in the test cohort (Appendix Table A5). Post-processing of multiresolution prediction maps and lymphocyte infiltration maps revealed that the fraction of lymphocyte-positive 
TABLE 3. AUC Performance of Clinicopathologic Logistic Regression, AI Score, and Combined Clinicopathologic + AI Score Models

Model Performance (AUC)

\begin{tabular}{|c|c|c|c|c|c|c|c|c|c|}
\hline \multirow[b]{2}{*}{ Cohort } & \multicolumn{2}{|c|}{$\begin{array}{c}\text { Clinicopathologic } \\
\text { Model }\end{array}$} & \multicolumn{2}{|r|}{ Al Score } & \multicolumn{2}{|c|}{$\begin{array}{l}\text { Clinicopathologic } \\
\text { Model + AI Score }\end{array}$} & \multicolumn{3}{|c|}{ Model Comparison $(P)$} \\
\hline & AUC & $95 \% \mathrm{CI}$ & AUC & $95 \% \mathrm{CI}$ & AUC & $95 \% \mathrm{Cl}$ & Clinical v AI & Clinical $v$ Clinical + Al & Al v Clinical + Al \\
\hline Test & 0.678 & 0.554 to 0.802 & 0.784 & 0.683 to 0.885 & 0.807 & 0.702 to 0.912 & .208 & .047 & .392 \\
\hline
\end{tabular}

NOTE. Significance testing of pairwise differences in area under the curve is reported, and $P<.05$ is accepted as significant.

Abbreviations: Al, artificial intelligence; AUC, area under the curve.

regions in the tumor area that were positive by the majority vote method was significantly lower than the fraction of lymphocyte-negative regions in tumor area that were negative by the majority vote method (Wilcoxon signed-rank $P=3.66 \times 10^{-8}$ ).

\section{DISCUSSION}

Recurrence rates remain high for patients undergoing radical cystectomy after diagnosis of localized MIBC, in which current prognostication and treatment selection remains highly dependent on tumor staging, grading, and the presence of variant histologic features. ${ }^{19}$ These features are often limited or inconsistently reported..$^{20}$ Thus, there is an urgent need for improved prognostication for patients with bladder cancer. In this study, we demonstrated the ability of deep learning algorithms to identify pathologic features within primary tumors associated with metastatic spread to LNs. Within this novel approach, we demonstrated that the combination of deep learning predictions with microenvironmental features results in a robust Albased method that significantly improves prognostication compared with clinicopathologic features. This Al approach is not subject to observer variability, which demonstrates consistent mapping to the risk of LN metastasis that generalized well to a leave-out test cohort of 88 patients from 22 centers.

Classification of tumors into risk groups based on histologic characterization is a key part of clinical risk stratification in multiple types of cancers such as Gleason scoring in prostate cancer or Fuhrman grading in clear cell renal cell carcinoma. ${ }^{21,22}$ Here, expert pathologists used experiencebased knowledge to define specific morphologic features to create risk groups, which are often subsequently retrospectively validated against patient outcomes. With our Al approach, CNNs learn the imaging characteristics of a tumor that are most strongly associated with LN spread. Current literature supports the notion that CNNs could have the capacity to learn unique features at each magnification
A

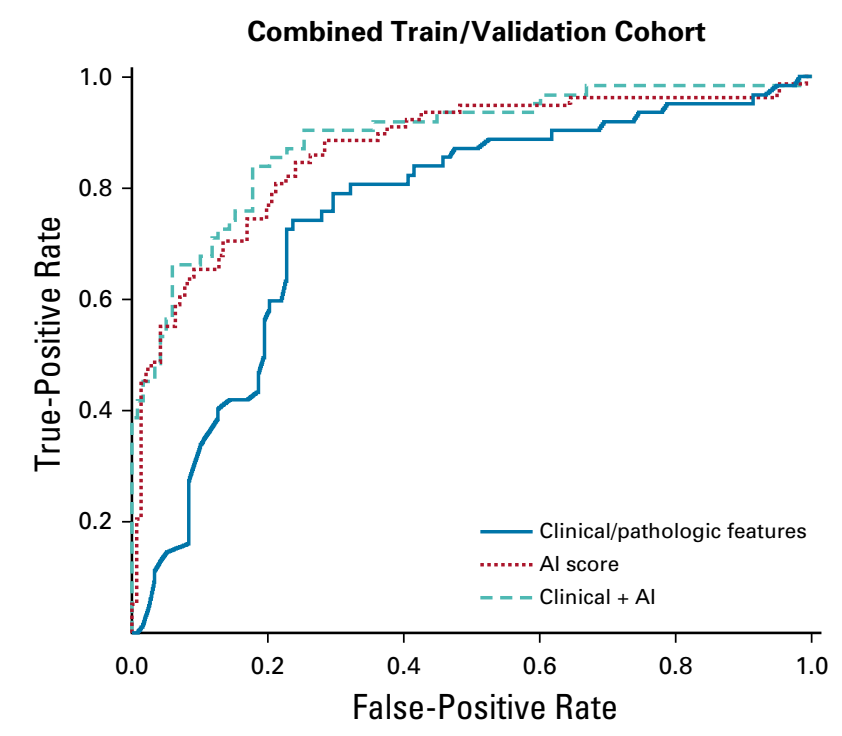

\section{B}

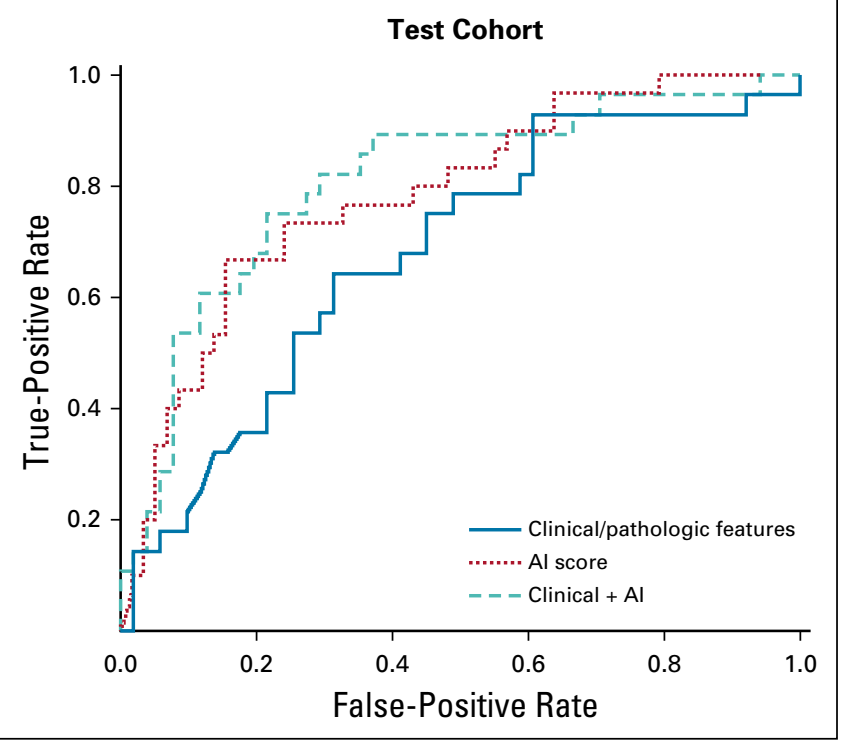

FIG 2. Receiver operating characteristic curves for $(A)$ combined training and validation cohorts and (B) test cohort. In the training and validation cohorts, artificial intelligence (AI) score is reported for out-of-bag predictions from 10-fold cross validation. In both cases, the clinicopathologic + Al model significantly outperformed the clinicopathologic model alone. 


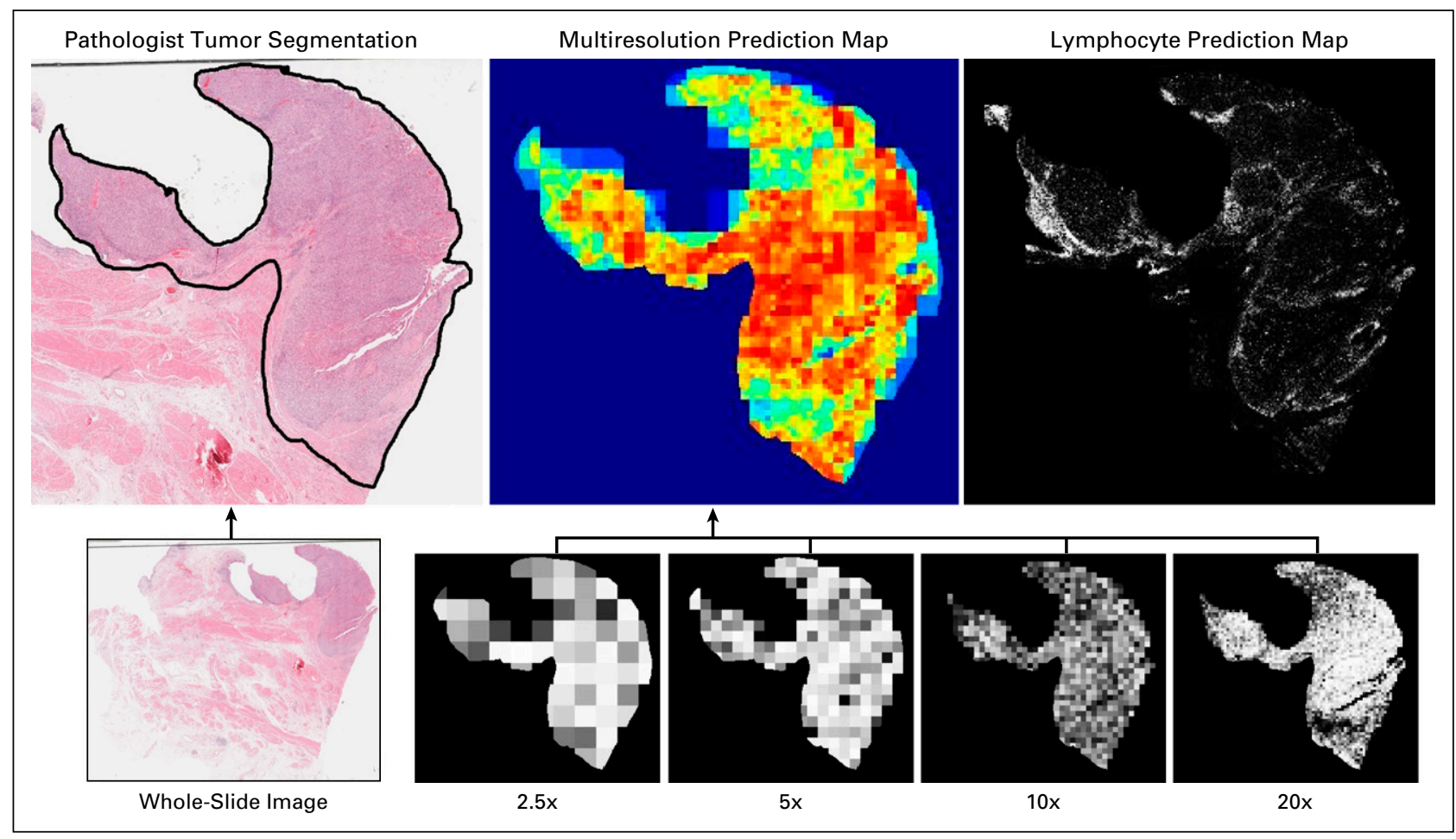

FIG 3. Representative example slides from a true-positive patient from an independent test set. Tumor regions from the whole slide image annotated by the pathologist were fed into each pretrained convolutional neural network for $2.5 \times, 5 \times, 10 x$, and $20 \times$ magnifications. All were averaged for a multiresolution prediction map and combined with a lymphocyte prediction map for machine learning-based classification.

level of digital pathology slides. ${ }^{23,24}$ Here, we trained separate models at multiple resolutions and spatially aggregated them to generate a tumor-level probability map. We found that the CNNs learned features known to be associated with poor prognosis, such as high-risk variant histologies. Given the improved prognostic performance of the $\mathrm{Al}$ approach over using standard clinicopathologic variables, we suggest that the Al approach learned unique features associated with the potential for $L N$ spread not reported or described in traditional clinical workflow. Furthermore, this approach achieved similar performance results when applied to an independent albeit small inhouse cohort.

A unique aspect of this study was the evaluation of tumorlevel prognosis in the setting of substantial intratumor heterogeneity. ${ }^{25}$ The multiresolution probability maps created from CNN outputs demonstrated heterogeneity in the distribution of high-risk features defined as high probability areas. Furthermore, the information learned directly from the H\&E slides was more predictive when combined with information from the tumor microenvironment (tumor-infiltrating lymphocytes). Of the twenty-six highly predictive features selected for use in the machine learning-based ensemble classifier, eight accounted for the presence and burden of infiltrating lymphocytes. The spatial distribution of tumor-infiltrating lymphocytes on $H \& E$ images was shown to have prognostic importance in multiple tumor types in the original publication of the algorithm used in this study. ${ }^{11}$ Independent studies that evaluated certain populations of lymphocytes by immunohistochemistry have already demonstrated more favorable prognosis in patients with high lymphocyte density at cystectomy. ${ }^{26,27}$ Taken together, our data demonstrate that the presence of any lymphocyte on H\&E staining (ie, not distinguished by phenotype) significantly improved discrimination of patient samples with high versus low metastatic potential when combined with output from deep learning-based algorithms, indicating that the complex effect of microenvironmental features on prognosis can be learned from Al-based systems.

The clinical application of this work may have an impact when applied to TURBT specimens for selecting patients most likely to benefit from NAC. A prospective trial of NAC + $R C$ versus $R C$ alone demonstrated improved survival for patients treated with NAC + RC. ${ }^{6}$ However, meta-analysis of NAC showed that the overall survival benefit for NAC was only 5\%. ${ }^{19}$ These trials were performed without substratifying patients with MIBC into risk groups; thus, the results of these trials may be diminished by the inclusion of low-risk patients who would not have developed recurrence with observation alone. To address the need for further stratification, previous studies have used clinical and pathologic variables to substratify MIBC into low-risk and high-risk groups and have proposed that low-risk patients may forego NAC. ${ }^{28}$ This strategy is based on the association between adverse outcomes and advanced clinical T stage, 
hydronephrosis, and adverse variant pathologic features (LVI, micropapillary features, or neuroendocrine features). ${ }^{28}$ However, clinical $T$ stage is frequently discordant with pathologic T stage, ${ }^{29,30}$ and the presence of variant features varies widely ( $7 \%$ to $81 \%$ ) among reported series. ${ }^{8}$ Validation of the approach used in this study in TURBT specimens may motivate physicians to use it as a prognostic marker in efforts to stratify patients before surgery.

This study has several important limitations. Although this methodology was developed in a heterogeneous data set, all of the specimens were cystectomy specimens. An independent test set was created to model evaluation, but it consisted mainly of TCGA patients because of the limited availability of patients from our own institution ( $n=13$ ). Within this in-house subset, accuracy remained high with 11 of 13 correctly classified. Deep learning algorithms are highly prone to overfitting, especially when trained on small data sets. Although our algorithms were trained on only 146 patients, each model was trained from thousands of patches per resolution (Appendix Table A1). We used techniques such as pretrained weights, stain normalization, and image augmentation to avoid overfitting. However, overall evaluation was still limited to patient-level analysis and therefore requires further validation in external populations.

\section{AFFILIATIONS}

${ }^{1}$ Molecular Imaging Branch, National Cancer Institute, Bethesda, MD ${ }^{2}$ Clinical Research Directorate, Frederick National Laboratory for Cancer Research, Frederick, MD

${ }^{3}$ Department of Urology, Upstate Medical University, Syracuse, NY ${ }^{4}$ National Library of Medicine, National Institutes of Health, Bethesda, MD

${ }^{5}$ Urologic Oncology Branch, National Cancer Institute, Bethesda, MD

${ }^{6}$ Division of Cancer Treatment and Diagnosis, Biometric Research

Program, National Cancer Institute, Bethesda, MD

\section{CORRESPONDING AUTHOR}

Baris Turkbey, PhD, National Cancer Institute, 9000 Rockville Pike, Bethesda, MD 20892; email: ismail.turkbey@nih.gov

\section{EQUAL CONTRIBUTION}

S.H. and T.H.S. contributed equally to this study.

\section{SUPPORT}

This project has been funded in whole or in part with federal funds from the National Cancer Institute, National Institutes of Health, under Contract No. HHSN261200800001E. The content of this publication does not necessarily reflect the views or policies of the Department of Health and Human Services, nor does mention of trade names, commercial products, or organizations imply endorsement by the U.S. Government.

\section{AUTHOR CONTRIBUTIONS}

Conception and design: Stephanie A. Harmon, Thomas H. Sanford, Sherif Mehralivand, Peter L. Choyke, Baris Turkbey

Provision of study materials or patients: Stephanie A. Harmon, Thomas $\mathrm{H}$. Sanford, Piyush K. Agarwal
Because of the variation in the number of available slides on TCGA, validation of the Al-based model in the TCGA testing cohort used only a single slide labeled DX1. In addition, some pathology slides had a substantial number of artifacts such as ink and dust, so they required preprocessing. Tumor heterogeneity could lead to incorrect classification of samples when evaluated across all tumor slides. Slide-level performance in the in-house cohort tracked similarly with patient-level performance. There was also a variable number of LNs sampled, which diminished the quality of the ground truth labels. Variability in TCGA clinical data led to missing data for LVI in $16 \%$ of the patients. Given the clinical importance of this pathologic feature, it is possible that the power of the clinical model may differ when compared with a complete set with nonmissing values.

In conclusion, we demonstrated a novel methodology for identifying patients who are at higher risk of having positive LNs during cystectomy based on digital H\&E slides from the primary tumor. This methodology is not subject to intra- or interobserver variation and can be generalized to multiple centers. All code used in developing this methodology is publicly available. This methodology may be useful for identifying patients most likely to benefit from additional therapy beyond surgical resection.

Collection and assembly of data: Stephanie A. Harmon, Thomas $\mathrm{H}$. Sanford, Chris Yang, Vladimir A. Valera, Piyush K. Agarwal, Baris Turkbey Data analysis and interpretation: Stephanie A. Harmon, Thomas $\mathrm{H}$.

Sanford, G. Thomas Brown, Joseph M. Jacob, Vladimir A. Valera, Joanna H. Shih, Peter L. Choyke, Baris Turkbey

Manuscript writing: All authors

Final approval of manuscript: All authors

Accountable for all aspects of the work: All authors

\section{AUTHORS' DISCLOSURES OF POTENTIAL CONFLICTS OF INTEREST}

The following represents disclosure information provided by authors of this manuscript. All relationships are considered compensated unless otherwise noted. Relationships are self-held unless noted. I = Immediate Family Member, Inst = My Institution. Relationships may not relate to the subject matter of this manuscript. For more information about ASCO's conflict of interest policy, please refer to www.asco.org/rwc or ascopubs. org/cci/author-center.

Open Payments is a public database containing information reported by companies about payments made to US-licensed physicians (Open Payments).

Stephanie A. Harmon

Patents, Royalties, Other Intellectual Property: Patent: System and Method for Evaluation of Disease Burden

\section{Sherif Mehralivand}

Stock and Other Ownership Interests: AbbVie

Piyush K. Agarwal

Employment: Pfizer (I)

Travel, Accommodations, Expenses: AstraZeneca/MedImmune 


\section{Peter L. Choyke}

Patents, Royalties, Other Intellectual Property: Patent holder for magnetic resonance imaging-ultrasound fusion technology licensed to InVivo which markets it as UroNav. However, as a government employee personally receive no financial benefit from this patent.

Other Relationship: Aspyrian Therapeutics, Philips Healthcare, GE Health Care

\section{Baris Turkbey}

Patents, Royalties, Other Intellectual Property: Royalties from US government patents for magnetic resonance imaging/ultrasound fusion biopsy computer-aided diagnosis software.

Other Relationship: NVIDIA, Philips Healthcare

No other potential conflicts of interest were reported.

\section{REFERENCES}

1. Millán-Rodríguez F, Chéchile-Toniolo G, Salvador-Bayarri J, et al: Primary superficial bladder cancer risk groups according to progression, mortality and recurrence. J Urol 164:680-684, 2000

2. Mari A, Campi R, Tellini R, et al: Patterns and predictors of recurrence after open radical cystectomy for bladder cancer: A comprehensive review of the literature. World J Urol 36:157-170, 2018

3. Gakis G, Efstathiou J, Lerner SP, et al: ICUD-EAU International Consultation on Bladder Cancer 2012: Radical cystectomy and bladder preservation for muscleinvasive urothelial carcinoma of the bladder. Eur Urol 63:45-57, 2013

4. Dotan ZA, Kavanagh K, Yossepowitch O, et al: Positive surgical margins in soft tissue following radical cystectomy for bladder cancer and cancer specific survival. J Urol 178:2308-2312, 2007

5. Bassi P, Ferrante GD, Piazza N, et al: Prognostic factors of outcome after radical cystectomy for bladder cancer: A retrospective study of a homogeneous patient cohort. J Urol 161:1494-1497, 1999

6. Grossman HB, Natale RB, Tangen CM, et al: Neoadjuvant chemotherapy plus cystectomy compared with cystectomy alone for locally advanced bladder cancer. N Engl J Med 349:859-866, 2003

7. Kluth LA, Black PC, Bochner BH, et al: Prognostic and prediction tools in bladder cancer: A comprehensive review of the literature. Eur Urol 68:238-253, 2015

8. Chalasani V, Chin JL, Izawa JI: Histologic variants of urothelial bladder cancer and nonurothelial histology in bladder cancer. Can Urol Assoc J 3:S193-S198, 2009

9. Bera K, Schalper KA, Rimm DL, et al: Artificial intelligence in digital pathology: New tools for diagnosis and precision oncology. Nat Rev Clin Oncol 16:703-715, 2019

10. Liu J, Lichtenberg T, Hoadley KA, et al: An integrated TCGA pan-cancer clinical data resource to drive high-quality survival outcome analytics. Cell 173:400-416.e11, 2018

11. Saltz J, Gupta R, Hou L, et al: Spatial organization and molecular correlation of tumor-infiltrating lymphocytes using deep learning on pathology images. Cell Rep 23:181-193.e7, 2018

12. Linkert M, Rueden CT, Allan C, et al: Metadata matters: Access to image data in the real world. J Cell Biol 189:777-782, 2010

13. Goldberg IG, Allan C, Burel JM, et al: The open microscopy environment (OME) data model and XML file: Open tools for informatics and quantitative analysis in biological imaging. Genome Biol 6:R47, 2005

14. Macenko M, Niethammer M, Marron JS, et al: A method for normalizing histology slides for quantitative analysis. 2009 IEEE International Symposium on Biomedical Imaging: From Nano to Macro, Boston, MA, June 28-July 1, 2009, 1107-1110

15. Ruifrok AC, Johnston DA: Quantification of histochemical staining by color deconvolution. Anal Quant Cytol Histol 23:291-299, 2001

16. Hou L, Nguyen V, Kanevsky AB, et al: Sparse autoencoder for unsupervised nucleus detection and representation in histopathology images. Pattern Recognit $86: 188-200,2019$

17. Loh WY: Regression trees with unbiased variable selection and interaction detection. Stat $\operatorname{Sin} 12: 361-386,2002$. http://pages.stat.wisc.edu/ loh/treeprogs/ guide/guide02.pdf

18. Friedman J, Hastie T, Tibshirani R: Additive logistic regression: A statistical view of boosting. Ann Stat 28:337-407, 2000

19. Veskimäe E, Espinos EL, Bruins HM, et al: What is the prognostic and clinical importance of urothelial and nonurothelial histological variants of bladder cancer in predicting oncological outcomes in patients with muscle-invasive and metastatic bladder cancer? A European Association of Urology Muscle Invasive and Metastatic Bladder Cancer Guidelines Panel Systematic Review. Eur Urol Oncol 2:625-642, 2019

20. Zhang L, Wu B, Zha Z, et al: Clinicopathological factors in bladder cancer for cancer-specific survival outcomes following radical cystectomy: A systematic review and meta-analysis. BMC Cancer 19:716, 2019

21. Epstein JI, Allsbrook WC Jr, Amin MB, et al: The 2005 International Society of Urological Pathology (ISUP) consensus conference on Gleason grading of prostatic carcinoma. Am J Surg Pathol 29:1228-1242, 2005

22. Fuhrman SA, Lasky LC, Limas C: Prognostic significance of morphologic parameters in renal cell carcinoma. Am J Surg Pathol 6:655-663, 1982

23. BenTaieb A, Li-Chang $\mathrm{H}$, Huntsman D, et al: A structured latent model for ovarian carcinoma subtyping from histopathology slides. Med Image Anal 39:194-205, 2017

24. Li J, Sarma KV, Chung Ho K, et al: A multi-scale U-Net for semantic segmentation of histological images from radical prostatectomies. AMIA Annu Symp Proc 2017:1140-1148, 2018

25. Warrick JI, Sjödahl G, Kaag M, et al: Intratumoral heterogeneity of bladder cancer by molecular subtypes and histologic variants. Eur Urol 75:18-22, 2019

26. Sjödahl G, Lövgren K, Lauss M, et al: Infiltration of $\mathrm{CD}^{+}$and $\mathrm{CD} 8^{+}$cells in bladder cancer is subtype specific and affects the outcome of patients with muscleinvasive tumors. Urol Oncol 32:791-797, 2014

27. Yu A, Mansure JJ, Solanki S, et al: Presence of lymphocytic infiltrate cytotoxic T lymphocyte CD3+, CD8+, and immunoscore as prognostic marker in patients after radical cystectomy. PLoS One 13:e0205746, 2018

28. Culp SH, Dickstein RJ, Grossman HB, et al: Refining patient selection for neoadjuvant chemotherapy before radical cystectomy. J Urol 191:40-47, 2014

29. von Rundstedt FC, Mata DA, Kryvenko ON, et al: Utility of clinical risk stratification in the selection of muscle-invasive bladder cancer patients for neoadjuvant chemotherapy: A retrospective cohort study. Bladder Cancer 3:35-44, 2017

30. Sherif A: The risk of oversimplification in risk-stratification of neoadjuvant chemotherapy-responses in muscle invasive bladder cancer. Transl Androl Urol 8:S337-S340, 2019 


\section{APPENDIX}

DATA SOURCE AND DOWNLOAD PROCEDURES FOR STUDY REPRODUCIBILITY

\section{Digital Pathology Images}

Central access site: portal.gdc.cancer.gov

- Select "repository"

- Under "File" Filter options select:

- Data Type $\rightarrow$ Slide Image

- Experimental Strategy $\rightarrow$ Diagnostic Slide

- Under "Cases" Filter options select Primary Site $\rightarrow$ Bladder

- Download manifest file

- Follow instructions from NCl GDC on downloading and using the GDC Transfer Tool: https://docs.gdc.cancer.gov/Data_Transfer_ Tool/Users_Guide/Getting_Started/

\section{Pathology Reports}

Central access site: https://www.cbioportal.org/

- Query $\rightarrow$ "Bladder/Urinary Tract" $\rightarrow$ "Bladder Cancer (TCGA, PanCancer Atlas)"

- Direct link to study: https://www.cbioportal.org/study/clinicalData? id=blca_tcga_pan_can_atlas_2018

- Individual patients can be searched/selected within "Clinical Data" Tab

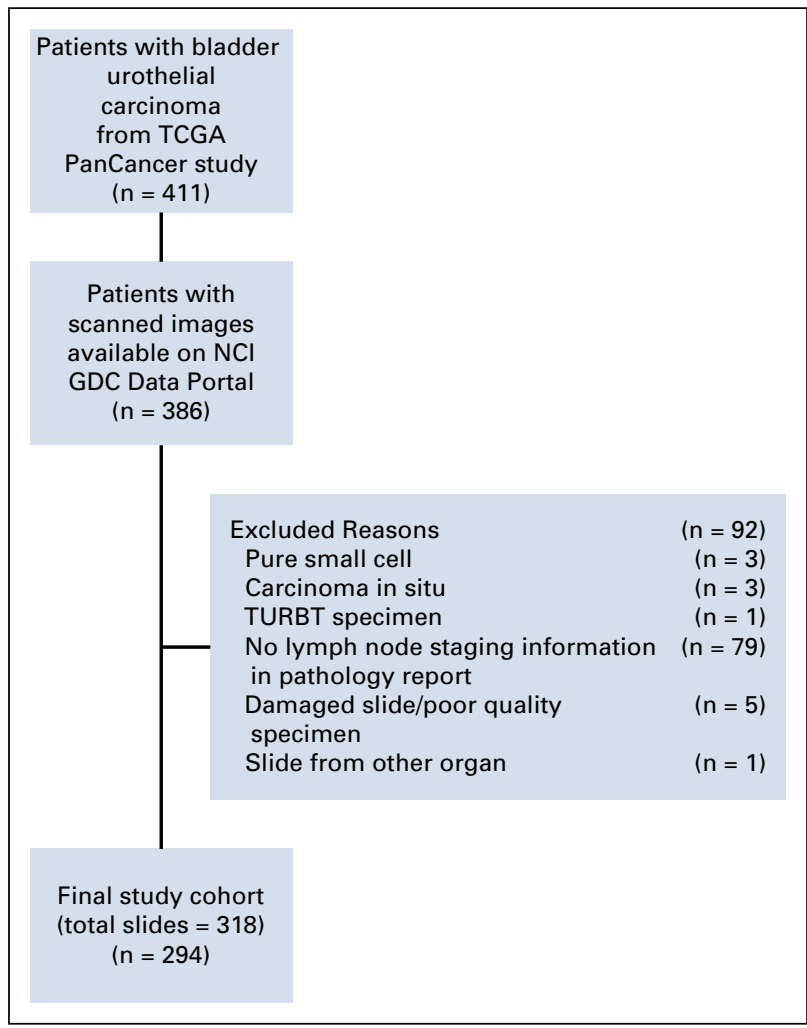

FIG A1. Final study selection. GDC, Genomic Data Commons; NCI, National Cancer Institute; TCGA, The Cancer Genome Atlas; TURBT, transurethral resection of bladder tumor.

\section{Clinical End Point Data}

Central access site: https://gdc.cancer.gov/about-data/publications/ pancanatlas

- Download file under "TCGA-Clinical Data Resource (CDR) Outcome"

- Corresponding publication: https://www.cell.com/cell/fulltext/S00928674(18)30229-0

\section{Patch Extraction and Stain Normalization}

For digital slides, images were read in at a 4:1 ratio from apparent 40x magnification (effective 10x magnification) digital images to allow for whole-slide image processing. ${ }^{13,14}$ Staining was normalized to an independent reference specimen not included in the data cohort using the Macenko method. ${ }^{7}$ Background artifacts and ink markings were removed and the background intensity was set before deconvolution of the 3-channel image using hematoxylin and eosin stains and the Ruifrok and Johnson method. ${ }^{8}$ All stain normalization and color deconvolution was implemented by using the Stain Normalization Toolbox for MATLAB (https://warwick.ac.uk/fac/sci/dcs/research/tia/ software/sntoolbox/).

All patches were derived in reference to $1,200 \times 1,200$ pixel regions at $10 \times$ magnification, with the number of regions determined by fitting the minimum number of non-overlapping regions that fully contained the pathologist's annotations. Within each of these reference regions, patch images were extracted at four separate image resolutions. The reference $1,200 \times 1,200$ region was resampled to a single $300 \times 300$ image ( $1 / 4$ original size) corresponding to effective $2.5 \times$ magnification, four $300 \times 300$ images ( $1 /$ 2 original size) corresponding to effective $5 \times$ magnification, and sixteen $300 \times 300$ images (no resampling) corresponding to $10 \times$ magnification. Coordinates of the reference region at $10 \times$ magnification were mapped to $40 \times$ magnification coordinates $(4,800 \times$ 4,800 pixels) to allow for extraction of sixty-four $300 \times 300$ images at 20x effective magnification, for which background artifacts and stain deconvolution were applied in reference to the corresponding region. The same coordinates were used to guide extraction of 576 images of $100 \times 100$ pixels at effective $20 \times$ resolution from the original (non-normalized, non-deconvolved) hematoxylin and eosin image for estimation of lymphocyte infiltration using the methods of Saltz et al. ${ }^{9,10}$

\section{Patch-Based Classification}

Four different models using ResNet-101 architecture were trained from patches at $2.5 \times, 5 \times, 10 \times$, and $20 \times$ magnifications. All patches were at an effective $300 \times 300$ image size from extraction and were labeled according to patient lymph node (LN) status. Patches containing less than $90 \%, 80 \%, 70 \%$, and $70 \%$ of white space (determined by proportion of normalized grayscale image $>0.8$ ) for $2.5 x$, $5 \times, 10 x$, and $20 x$ magnifications, respectively, were considered for input. A weighted cross-entropy loss was applied at the time of patchlevel training to offset bias from the observed class imbalance at the patient level. Data augmentation techniques, including image rotation, flipping, warping, brightness, and contrast were applied randomly at training time to reduce overfitting to patient-specific and slide-specific characteristics. The final training parameters, including number of epochs, learning rate, imbalance technique, and data augmentation scheme, are included in Appendix Table A1. All models were trained using fast.ai library (https://github.com/fastai/fastai). After training was completed, all models were deployed to all patches within training, validation, and testing cohorts and the probability of being LN positive was saved for each patch image at every resolution. For lymphocyte classification, publicly available code and model weights were downloaded from Saltz et al. ${ }^{9}$ These models were applied to all $100 \times$ 100 pixel images from all patient samples extracted during preprocessing, and the probability of containing lymphocytes was saved for each patch. Consistency in lymphocyte maps derived from the preprocessing methods in this study versus the original study were confirmed (Appendix Fig A2). 


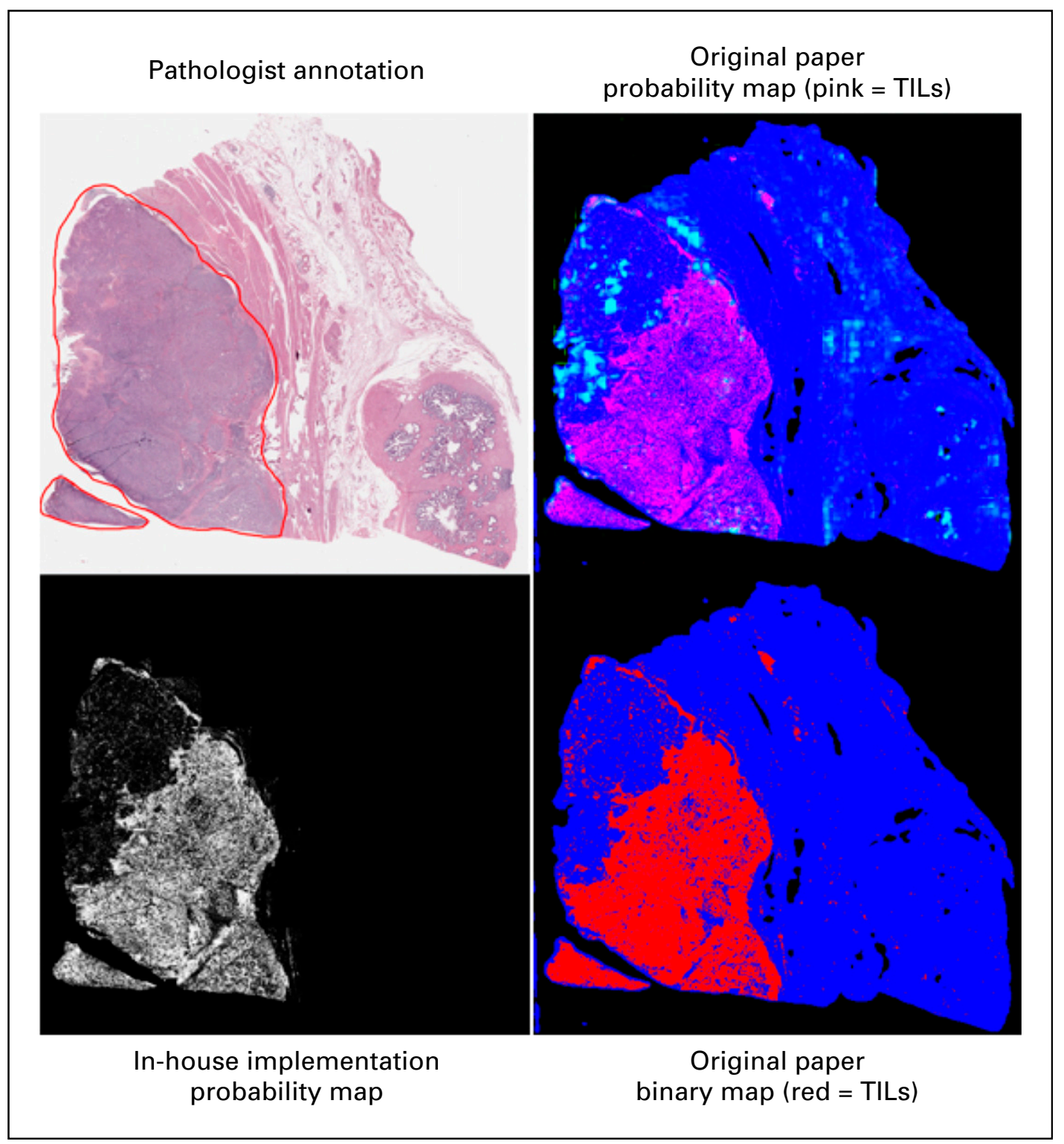

FIG A2. Representative lymphocyte map from in-house implementation $v$ the original publication. TILs, tumor-infiltrating lymphocytes.

\section{Post-Processing and Patient-Based Classification}

Given that patches at all resolutions were derived spatially within known coordinates from the original image, a multiresolution prediction map was derived from all LN status classification and lymphocyte classification results. The final prediction maps were created at $1 / 200$ the size of original $40 \times$ apparent magnification image, such that each lymphocyte prediction corresponded to a single pixel, each effective $20 \times$ prediction corresponded to $3 \times 3$ pixel area, each $10 \times$ prediction corresponded to a $6 \times 6$ pixel area, each $5 \times$ prediction corresponded to a $12 \times 12$ pixel area, and each $2.5 \times$ prediction corresponded to a $24 \times 24$ pixel area. Lymphocyte prediction maps were derived separately from LN classification maps. For each LN classification, maps of each resolution and per-pixel average prediction across all resolutions were created. The final result is an image in which every pixel represents the regional probability of LN status.

From the resultant pixel-based probability maps, high-risk clusters (ie, high probability of LN positivity by classification algorithms) were identified by using density-based spatial clustering of applications with noise (DBSCAN) implementation in MATLAB. Twelve different starting conditions derived from varying threshold probability cutoffs within various combinations of multiresolution probability maps. For each condition, metrics related to the size, number, density, and distance between clusters were extracted. In addition, distance between each resultant cluster and lymphocyte-rich pixels was tabulated. Finally, overall average probability of each resolution and total average across all resolutions were calculated for each patient. In total, 716 elements were extracted from representative slides for each patient in all cohorts. A full description of probability map derivation, DBSCAN parameters, and extracted features is provided in Appendix Table A2.

For image-based classification, an adaptive boosting classification ensemble of decision trees (AdaBoost) was fit using 10-fold cross validation within combined training and validation cohorts. Feature selection was determined during each partition on the basis of predictor importance estimates from decision trees using all variables. Within each partition, the top 20 important features were selected, resulting in 40 unique variables selected across $10-$ folds. Any variables selected as a top feature in more than one partition were considered for 
TABLE A1. Patch-Based Deep Learning Training Schemes and Performance

Training Schemes

TCGA Patch-Based

Performance

\begin{tabular}{|c|c|c|c|c|c|c|c|c|c|c|c|}
\hline \multirow[b]{2}{*}{ Magnification } & \multicolumn{2}{|c|}{$\begin{array}{c}\text { No. of Training } \\
\text { Patches }\end{array}$} & \multirow[b]{2}{*}{$\begin{array}{c}\text { White } \\
\text { Restriction }\end{array}$} & \multirow[b]{2}{*}{$\begin{array}{l}\text { No. of Training } \\
\text { Epochs }\end{array}$} & \multirow[b]{2}{*}{$\begin{array}{l}\text { Batch } \\
\text { Size }\end{array}$} & \multirow[b]{2}{*}{$\begin{array}{l}\text { Learning } \\
\text { Rate }\end{array}$} & \multirow[b]{2}{*}{$\begin{array}{l}\text { Weighted Loss } \\
\text { Negative:Positive }\end{array}$} & \multicolumn{2}{|c|}{$\begin{array}{l}\text { Validation } \\
\text { Cohort (\%) }\end{array}$} & \multicolumn{2}{|c|}{$\begin{array}{c}\text { Test Cohort } \\
(\%)\end{array}$} \\
\hline & $\begin{array}{l}\text { Negative } \\
\text { Class }\end{array}$ & $\begin{array}{l}\text { Positive } \\
\text { Class }\end{array}$ & & & & & & ACC & F1 & ACC & F1 \\
\hline $2.5 x$ & 11,875 & 6,677 & 90 & 3 & 45 & $3.0 \times 10^{-5}$ & $1: 1.05$ & 66.7 & 44.6 & 64.4 & 56.4 \\
\hline $5 x$ & 43,961 & 24,919 & 80 & 50 & 45 & $1.0 \times 10^{-6}$ & $1.05: 1$ & 64.5 & 50.1 & 62.7 & 53.5 \\
\hline $10 x$ & 168,586 & 95,964 & 70 & 3 & 32 & $8.0 \times 10^{-6}$ & $1.75: 1$ & 68.7 & 39.1 & 61.9 & 35.6 \\
\hline $20 x$ & 664,754 & 379,404 & 70 & 3 & 32 & $1.0 \times 10^{-6}$ & $2: 1$ & 63.1 & 27.9 & 58.8 & 29.9 \\
\hline
\end{tabular}

NOTE. For training of patch-based classifiers, each patch was assigned a label based on the lymph node status of the patient. Abbreviations: ACC, accuracy; F1, F1 score; TCGA, The Cancer Genome Atlas.

final model inclusion $(n=26)$, in which the final model was trained by repeating 10 -fold cross validation selecting 15 features using an interaction test for variable selection. ${ }^{11}$ The final model was trained by using a maximum of three splits, a learning rate of $0.001,4,000$ learning cycles, and a 1.382 misclassification cost for false positives. Classification performance was determined by out-of-bag estimates during cross validation. The final model was then applied to testing cohorts. Classification outputs were saved as binary (yes/no for LN positive) and continuous (classification fit: range, 0-1 probability of LN positivity), which is referred to as the artificial intelligence (AI) score. The image-based classifier was developed in MATLAB (R2018b, www. mathworks.com) using Statistics and Machine Learning Toolbox. 
TABLE A2. Features Selection for Use in Machine Learning Classifier

Source Image

Threshold

\begin{tabular}{|c|c|c|}
\hline Average $(2.5 x+5 x+10 x+20 x)$ & 0.4 & Total cluster area/tumor area \\
\hline Average $(2.5 x+5 x+10 x+20 x)$ & 0.5 & Total cluster size \\
\hline Average $(2.5 x+5 x+10 x+20 x)$ & 0.5 & Total cluster area/tumor area \\
\hline Average $(2.5 x+5 x+10 x+20 x)$ & 0.6 & Total cluster size \\
\hline Average $(2.5 x+5 x+10 x+20 x)$ & 0.6 & Total cluster area/tumor area \\
\hline Product $(2.5 x+5 x+10 x+20 x)$ & 0.05 & Total cluster size \\
\hline Binary $(2.5 x+5 x+10 x)$ & 1 & Total cluster area/tumor area \\
\hline Binary $(2.5 x+5 x+10 x)$ & 2 & Total cluster size \\
\hline Binary $(2.5 x+5 x+10 x)$ & 2 & Total cluster area/tumor area \\
\hline Average $(2.5 x+5 x+10 x)$ & 0.4 & Total cluster area/tumor area \\
\hline Average $(2.5 x+5 x+10 x)$ & 0.4 & Median probability for all clusters \\
\hline Average $(2.5 x+5 x+10 x)$ & 0.5 & Total cluster area/tumor area \\
\hline Average $(2.5 x+5 x+10 x)$ & 0.5 & Maximum cluster median \\
\hline Average $(2.5 x+5 x+10 x)$ & 0.5 & Median probability for all clusters \\
\hline Average $(2.5 x+5 x+10 x)$ & 0.6 & Total cluster area/tumor area \\
\hline Binary $(2.5 x+5 x+10 x)+$ TIL & 0.5 & Maximum cluster median \\
\hline Binary $(2.5 x+5 x+10 x)+$ TIL & 0.5 & Median probability for all clusters \\
\hline Binary $(2.5 x+5 x+10 x)+$ TIL & 0.5 & Average probability across all clusters \\
\hline Average $(2.5 x+5 x+10 x)+$ TIL & 0.5 & Total cluster area/tumor area \\
\hline Average $(2.5 x+5 x+10 x)+$ TIL & 0.75 & Total cluster area/tumor area \\
\hline Average $(2.5 x+5 x+10 x)+$ TIL & 0.75 & Maximum cluster median \\
\hline Average $(2.5 x+5 x+10 x)+$ TIL & 0.75 & Median probability for all clusters \\
\hline Average $(2.5 x+5 x+10 x+20 x) v$ TIL & 0.4 & Burden $(\mathrm{Al})+$ burden $(\mathrm{TIL})$ \\
\hline
\end{tabular}

Abbreviations: Al, artificial intelligence; TIL, tumor-infiltrating lymphocytes. 
TABLE A3. $\chi^{2}$ Test for Differences in Clinical and Pathologic Demographics of Training and Validation Versus Testing Cohorts

\begin{tabular}{|c|c|c|c|}
\hline Characteristic & $\begin{array}{c}\text { Training and } \\
\text { Validation Cohorts }\end{array}$ & $\begin{array}{c}\text { Test Cohort } \\
\text { (TCGA + in-house) }\end{array}$ & $\begin{array}{c}\chi^{2} \text { Test } \\
P \\
\end{array}$ \\
\hline Sex & & & .9012 \\
\hline Male & 161 & 66 & \\
\hline Female & 58 & 22 & \\
\hline Race & & & .5151 \\
\hline White & 180 & 63 & \\
\hline African American & 11 & 6 & \\
\hline Asian & 23 & 6 & \\
\hline Histologic grade & & & .3563 \\
\hline Low & 12 & 2 & \\
\hline High & 206 & 86 & \\
\hline \multicolumn{4}{|l|}{ Unknown } \\
\hline Smoking history & & & .7514 \\
\hline Lifelong nonsmoker & 61 & 27 & \\
\hline Current smoker & 45 & 15 & \\
\hline Reformed smoker & 105 & 41 & \\
\hline Pathologic $T$ stage & & & .7189 \\
\hline $\mathrm{T} 1 / \mathrm{T} 2$ & 66 & 24 & \\
\hline T3/T4 & 153 & 64 & \\
\hline High-risk variant histology & & & .7563 \\
\hline Not present & 208 & 85 & \\
\hline Present & 11 & 3 & \\
\hline LVI & & & .3632 \\
\hline Not present & 74 & 38 & \\
\hline Present & 106 & 41 & \\
\hline
\end{tabular}

Abbreviations: LVI, lymphovascular invasion; TCGA, The Cancer Genome Atlas.

TABLE A4. Machine Learning Classifier Performance in Training and Validation Versus Testing Cohorts

\begin{tabular}{|c|c|c|c|c|c|c|c|c|}
\hline Cohort & $\operatorname{ACC}(\%)$ & SENS (\%) & SPEC (\%) & PPV (\%) & TP & TN & FP & FN \\
\hline Training and validation (out-of-bag predictions) & 77.5 & 70.5 & 82.6 & 71.4 & 55 & 119 & 22 & 23 \\
\hline Test (TCGA + in-house) & 76.1 & 60 & 84.5 & 66.7 & 18 & 49 & 9 & 12 \\
\hline TCGA only & 74.7 & 63.0 & 81.3 & 65.4 & 17 & 39 & 9 & 10 \\
\hline In-house only & 84.6 & 33.3 & 100 & 100 & 1 & 10 & 0 & 2 \\
\hline Test (in-house only), by slide & 71.1 & 10 & 96.3 & 90.9 & 1 & 26 & 1 & 10 \\
\hline
\end{tabular}

NOTE. All performance metrics for the training and validation cohorts are reported for out-of-bag predictions from 10-fold cross validation. Testing cohorts are further broken down into The Cancer Genome Atlas (TCGA) $v$ in-house samples, which are subsequently reported as patient-level and slide-level metrics.

Abbreviations: ACC, accuracy; FN, false negatives; FP, false positives; PPV, positive predictive value; SENS, sensitivity; SPEC, specificity; TN, true negatives; TP, true positives. 
Harmon et al

TABLE A5. Machine Learning Performance of Simple Al $v$ Final Al Score

Model Performance (AUC)

\begin{tabular}{|c|c|c|c|c|c|}
\hline \multirow[b]{2}{*}{ Cohort } & \multicolumn{2}{|c|}{ Simplified Al } & \multicolumn{2}{|c|}{ Final Al } & \multirow{2}{*}{$\begin{array}{l}\text { Model Comparison } P \\
\quad \text { (clinical } v \mathrm{DL} \text { ) }\end{array}$} \\
\hline & Score & $95 \% \mathrm{Cl}$ & Score & $95 \% \mathrm{CI}$ & \\
\hline Training/validation & 0.8660 & 0.815 to 0.917 & 0.8664 & 0.812 to 0.920 & .968 \\
\hline Test & 0.7621 & 0.662 to 0.862 & 0.7839 & 0.702 to 0.896 & .240 \\
\hline
\end{tabular}

NOTE. Simplified Al score is determined as the burden of tumor with probability greater than $50 \%$ for lymph node positivity separately in $2.5 \times$, $5 \times$, and 10x models, also referred to as the majority-vote method.

Abbreviations: Al, artificial intelligence; AUC, area under the curve; DL, deep learning. 